\title{
Weakly 1-Complete Manifold and Levi Problem
}

\author{
By \\ Takeo OHSAWA*
}

\section{§ 0. Introduction}

Let $X$ be a paracompact complex manifold of dimension $n \geqq 1$. We call $X$ a weakly 1 -complete manifold if there exists a $C^{\infty}$ plurisubharmonic function $\varphi$ on $X$ such that for every $c \in \mathbb{R}$ (real number), $\varphi^{-1}((-\infty$, c)) is relatively compact in $X . \varphi$ is called an exhaustion function of $X$. It is well known that holomorphically convex manifolds are weakly 1-complete. The converse is not true in general (cf. [1]), so one is led to the problem of seeking natural additional conditions which make weakly 1-complete manifolds holomorphically convex.

The content of this article is divided into two parts; Section 1 is devoted to prove some properties of weakly 1 -complete manifolds which have a nonconstant holomorphic function. In Section 2, first we present an application of the Nakano's vanishing theorem to the Levi problem on projective spaces and hyperquadrics. These results are not new (cl. [3]) but the method will be of some interest. Next, combining the Nakano's vanishing theorem with the result in Section 1 and a well known theorem (due to Bonnet) of differential geometry we obtain the following.

Theorem 2. 2. Let $X$ be a weakly 1-complete manifold of dimension 2. If the canonical bundle of $X$ is negative, then $X$ is holomorphically convex.

The last paragraph is a variant of Section 2,1. Combining Theorem 2.2 with the Nakano's vanishing theorem we solve the Levi problem on

Received November 17, 1979. Revised April 26, 1980.

* Research Institute for Mathematical Sciences, Kyoto University, Kyoto 606, Japan. 
some hypersurfaces and complete intersections.

The author thanks Messrs. A. Takeuchi, A. Fujiki, Te. Ueda, and K. Takegoshi. The discussions with them led the author to this problem. He also thanks the referee for valuable criticisms. The proofs of Theorem 1. 1 and Proposition 1.4 are partly simplified by him (or her).

\section{§ 1. Weakly 1-Complete Manifold with a Nonconstant Holomorphic Function}

Let $X$ be a weakly 1 -complete manifold and $\varphi$ an exhaustion function on $X$. For a real number $c$ we denote by $X_{c}$ the sublevel set $\varphi^{-1}((-\infty, c))$. By the definition of $\varphi, X_{c}$ is relatively compact in $X$. We denote by $\partial X_{c}$ the (topological) boundary of $X_{c}$ in $X$.

Theorem 1. 1. Let $X$ be a connected reakly 1-complete manifold. If there exists a nonconstant holomorphic function $f: X \rightarrow \mathbb{C}$, then either.

i) $f^{-1}(z) \cap X_{c}$ is empty or noncompact for any $z \in \mathbb{C}$ and $c \in \mathbb{R}$. or

ii) $f^{-1}(z) \cap X_{c}$ is compact for any $z \in \mathbf{C}$ and $c \in \mathbf{R}$.

Proof. For a complex manifold $M$ and a holomorphic function $g$ on $M$, we denote by $F_{x}^{M}$ the connected component of $g^{-1}(g(x))$ that contains $x$. We need the following

Sublemma 1.2. Let $\Omega$ be an open set of $\mathbb{C}^{n}$ containing the origin $(0, \cdots, 0)$ and $f$ a holomorphic function on $\Omega$ such that $f(0, \cdots, 0)$ $=0$. Then there exists a neighbourhood $U$ of $(0, \cdots, 0)$ in $\Omega$ such that if $x_{k} \in U \backslash f^{-1}(0) \quad(k=1,2, \cdots)$ and $f\left(x_{k}\right) \rightarrow 0 \quad(k \rightarrow \infty)$, then $F_{x_{k}}^{U}$ are nonsingular and $\left.\operatorname{dist}\left(F_{x_{k}}^{U}, f^{-1}(0) \cap U\right)\right) \rightarrow 0 \quad(k \rightarrow \infty)$. Here $\operatorname{dist}(A, B)$ $:=\sup _{x \in \boldsymbol{A}} \inf _{y \in \boldsymbol{B}}|x-y|+\sup _{y \in \boldsymbol{B}} \inf _{\boldsymbol{x} \in \boldsymbol{A}}|y-x|$.

Proof of Sublemma 1.2. By the local parametrization theorem (cf. [2] p. 98, 10. Theorem), after a suitable change of coordinates we can choose a polydisc $d_{r}$ in $\mathbf{C}^{n}$ with radius $r=\left(r_{1}, \cdots, r_{n}\right)$ and center $(0, \cdots, 0)$ 
such that the projection $\pi$ defined by $\pi\left(z_{1}, \cdots, z_{n}\right)=\left(z_{1}, \cdots, z_{n-1}\right)$ induces proper holomorphic maps from $f^{-1}(0) \cap d_{r}$ and $f^{-1}\left(x_{k}\right) \cap d_{r}$ to $d_{r^{\prime}}$, where $r^{\prime}:=\left(r_{1}, \cdots, r_{n-1}\right)$. Let $l$ be a complex line in $\mathbf{C}^{n-1}$ through the origin. Then $\pi^{-1}(l)$ meets every irreducible component of $f^{-1}(0)$ which contains the origin. Therefore, in order to prove the sublemma we have only to prove the case $n=2$. Let $d_{r}$ be such that for any $x \in d_{r} \backslash f^{-1}(0)$, $\left.d f\right|_{x} \neq 0$ and $f^{-1}(0) \cap \Delta_{r}$ is connected. If $f^{-1}(0) \cap \Delta_{r}$ has irreducible components $V_{1}, \cdots, V_{m}$, there exists $\varepsilon>0$ such that $\{x ;|f(x)|<\varepsilon\} \cap \Delta_{r} \backslash \bar{\Delta}_{r / 2}$ has $m$ connected components $W_{1}, \cdots, W_{m}$ and $|f(x)|>\varepsilon$ if $\left|z_{2}\right|=r_{2}$. We let

$$
U_{i}:=\left\{x \in \Delta_{r} \cap\{x ;|f(x)|<\varepsilon\} \backslash f^{-1}(0) ; F_{x}^{\Delta_{r}} \cap\left(\Delta_{r} \backslash \bar{\Delta}_{r / 2}\right) \cap W_{i}=\emptyset\right\} .
$$

Clearly $U_{i}$ is open and closed in $d_{r} \cap\{x ;|f(x)|<\varepsilon\} \backslash f^{-1}(0)$. Assume $U_{i} \neq \emptyset$ for some $i$. Since $d_{r} \cap\{x ;|f(x)|<\varepsilon\} \backslash f^{-1}(0)$ is connected, it follows that $U_{i}=A_{r} \cap\{x ;|f(x)|<\varepsilon\} \backslash f^{-1}(0)$. This contradicts the definition of $U_{i}$. Thus $U_{i}=\emptyset$ for every $i$, whence the sublemma follows.

Proof of Theorem 1.1. We set

$$
B:=\left\{x \in X ; F_{x}^{X} \text { is compact }\right\} .
$$

To prove the theorem we have only to show that there are only two possibilities, i.e., $B$ is empty or $B=X$. This is equivalent to saying that $B$ is open and closed. It is easy to see that $B$ is open. In order to see that $B$ is closed, first we note that $F_{x}$ is compact if and only if $\left.\varphi\right|_{F_{x}}$ is constant. In fact, "if" part follows from the fact that $\varphi$ is an exhaustion function and "only if" part follows from that $\varphi$ is plurisubharmonic.

Suppose that $B$ is not closed. Then there exist a point $x_{0}$ in $X$ and a sequence of points $x_{k} \in B, k=1,2, \cdots$, such that $x_{k} \rightarrow x_{0}(k \rightarrow \infty)$ and $F_{x_{0}}$ is not compact. In virtue of the sublemma, $F_{x_{k}}$ converge to $F_{x_{0}}$ uniformly in a neighbourhood of $x_{0}$. Since $F_{x_{k}}$ are compact and contained in a compact subset of $X$ it follows that $F_{x_{k}}$ converge uniformly to $F_{x_{\mathrm{p}}}$. Therefore $\varphi$ must be constant on $F_{r_{0}}$. This is a contradiction.

Q.E.D.

Remark. We clid not use in the proof the differentiability of $\varphi$. 
Only the continuity of $\varphi$ suffices.

For the later use we quote here a theorem of R. Narashimhan.

Theorem 1.3 (cf. [6], Corollary 1). A weakly l-complete manifold $X$ is holomorphically convex if and only if $X_{c}$ is holomorphically convex for every $c \in \mathbf{R}$.

As a corollary to Theorem 1.1 we obtain

Proposition 1. 4.*) Let $X$ be a noncompact weakly 1-complete manifold of dimension 2. Then $X$ is holomorphically convex if and only if $X$ has a nonconstant holomorphic function.

Proof. The only if part is trivial. Let $f: X \rightarrow \mathbf{C}$ be a nonconstant holomorphic function. Then i) or ii) of Theorem 1.1 holds. If ii) holds then by Stein factorization theorem there exists an open Riemann surface $\mathscr{R}$ and a proper holomorphic map $g: X \rightarrow \mathscr{R}$. Since $\mathscr{R}$ is holomorphically convex (cf. [2]), $X$ is also holomorphically convex. If i) holds, then since the critical values of $\left.f\right|_{X_{c}}$ are finitely many and $f$ is constant on every compact connected subvariety of $X, X_{c}$ contains only finitely many compact irreducible curves. Therefore there exists $c^{\prime}<c$ such that $X_{c^{\prime}}$ contains all the compact curves contained in $X_{c}$. Let the connected components of these compact curves be denoted by $A_{i}, i=1, \cdots, m$. Let $U_{i}$ be a neighbourhood of $A_{i}$ such that $\bar{U}_{i} \subset X_{c^{\prime}}$. Then for every $z \in \mathbf{C}, f^{-1}(z) \cap X_{c^{\prime}}-\bigcup_{i=1}^{m} \bar{U}_{i}$ is a disjoint union of open Riemann surfaces.

Thus combining the theorem of Richberg (cf. [8], Satz 3.3) and the usual patching technique (cf. [9], Corollary 4.14) we obtain a strictly plurisubharmonic function $\psi$ defined on a neighbourhood of $\bar{X}_{c^{\prime \prime}}-\bigcup_{i=1}^{m} U_{i}$, where $c^{\prime \prime}$ is such that $X_{c^{\prime \prime}} \subset X_{c^{\prime}}$ and $X_{c^{\prime \prime}} \supset \bar{U}_{i}$ for any $i$. Therefore $X_{c^{\prime \prime}}$ is a strongly pseudoconvex domain in $X$. Since $c$ was arbitrary, by Theorem 1.3 we conclude that $X$ is holomorphically convex. Q.E.D.

$\therefore$ Proposition 1.4 can be viewed as a special case of the Theorem of Knorr and Schneider (cf. [4]). 


\section{§ 2. Levi Problem}

\section{The Levi problem on complex projective space and hyperquadrics}

Let $M$ and $N$ be complex manifolds. We say that $M$ is a domain over $N$ if there exists a holomorphic map $\pi: M \rightarrow N$ which is a local homeomorphism. Let $p: \mathbf{L} \rightarrow M I$ be a holomorphic line bundle over $M$ with trivializing covering $\left\{U_{i}\right\}$ and transition functions $\left\{e_{i j}\right\} . \mathbb{L}$ is called positive (semipositive) if there exists a metric $\left\{a_{i}\right\}$ along the fibers of $\mathbb{L}$ (i.e., $a_{i}$ and positive $C^{\infty}$ functions on $U_{i}$ satisfying $a_{i}\left|e_{i j}\right|^{2}=a_{j}$ on $U_{i} \cap U_{j}$ ) such that $-\sqrt{-1} \partial \bar{\partial} \log a_{i}$ is a positive (resp. semipositive) $(1,1)$ form on $M$. We say $\mathbf{L}$ is negative (seminegative) if the dual of $\mathbb{L}$ is positive (resp. semipositive).

We denote by $\mathbf{P}^{n}$ a complex projective space of dimension $n$ and by $\mathbb{Q}^{n}$ a complex hyperquadric in $\mathbf{P}^{n+1}$. We also denote by $\mathbb{K}_{X}$ a canonical bundle of $X$.

The following theorem was proved by A. Hirschowitz [3] under a less restrictive assumption but we present the proof here for the sake of its simplicity.

Theorem 2. 1. Let $X$ be a reakly 1-complete manifold. If $\mathrm{Y}$ is a domain over $\mathbf{P}^{n}$ or $\mathbf{Q}^{n}$, then $X$ is holomorphically convex.

Proof. We denote by $H^{n-1}$ a hyperplane in $\mathbf{P}^{n}$. We have

$$
\mathbb{K}_{\mathbf{p}^{n}}=\left[-(n+1) H^{n-1}\right]
$$

and

$$
\mathbf{K}_{\mathbf{Q}^{n}}=\left[-n\left(\left.H^{n}\right|_{\mathbf{Q}^{n}}\right)\right],
$$

where we denote by $[D]$ the line bundle associated to a divisor $D$.

First we prove the theorem for $\mathbb{P}^{n}$ by induction on $n$. For $n=1$, the theorem is well known (cf. [2]). Let us assume that we have proved the theorem for $n \leqq r,(r \geqq 1)$ and let $X$ be a weakly 1 -complete manifold which is a domain over $\mathbf{P}^{r+1}$. We are going to prove that $X_{c}$ is holomorphically convex for every $c \in \mathbb{R}$. If $X_{c}$ is compact then $X_{c}$ is 
trivially holomorphically convex. So we may assume $\partial X_{c} \neq \emptyset$. Let $x \in \partial X_{c}$ and $\pi: X \rightarrow \mathbf{P}^{r+1}$ be the given local homeomorphism. Let $y \in X_{c}$ and choose a hyperplane $H \subset \mathbf{P}^{r+1}$ which contains $\pi(x)$ and $\pi(y)$. Then $\pi^{-1}(H)$ is a noncompact submanifold of $X$ and by the induction hypothesis holomorphically convex. Therefore $\pi^{-1}(H) \cap X_{c}$ is also holomorphically convex. Hence, for any sequence $\left\{x_{k}\right\}(k=1,2, \cdots)$ satisfying $x_{k} \in X_{c}$ and $\lim _{k \rightarrow \infty} x_{k}=x$ there exists a holomorphic function $f$ on $\pi^{-1}(H) \cap X_{c}$ such that

$$
\sup _{k=1,2, \ldots}\left|f\left(x_{k}\right)\right|=\infty
$$

As usual the obstruction for finding a holomorphic function $\tilde{f}$ on $X_{c}$ such that $\left.\tilde{f}\right|_{\pi^{-1}(H) \cap X_{c}}=f$ lies in the cohomology group $H^{1}\left(X_{c},\left[\pi^{-1}(H)\right.\right.$ $\left.\left.\cap X_{c}\right]^{*}\right)$, where $\left[\pi^{-1}(H) \cap X_{c}\right]^{*}$ denotes the dual of the line bundle $\left[\pi^{-1}(H) \cap X_{c}\right]$. We are going to prove that this group is 0 . First,

$$
H^{1}\left(X_{c},\left[\pi^{-1}(H) \cap X_{c}\right]^{*}\right)=H^{1}\left(X_{c}, \mathbf{K}_{X_{c}} \otimes\left(\mathbf{K}_{X_{c}}^{*} \otimes\left[\pi^{-1}(H) \cap X_{c}\right]^{*}\right)\right),
$$

where $\mathbf{K}_{X_{c}}$ denotes the canonical bundle of $X_{c}$. Since

$$
\begin{aligned}
\mathbf{K}_{X_{\mathrm{c}}} & =\left(\left.\pi\right|_{X_{c}}\right) * \mathbf{K}_{\mathbf{p}^{r+1}}=\left(\left.\pi\right|_{X_{\mathrm{c}}}\right) *[-(r+2) H] \\
& =\left[(r+2) \pi^{-1}(H) \cap X_{c}\right]^{*}
\end{aligned}
$$

we have

$$
\begin{aligned}
& H^{1}\left(X_{c}, \mathbf{K}_{X_{c}} \otimes\left(\mathbf{K}_{X_{c}}^{*} \otimes\left[\pi^{-1}(H) \cap X_{c}\right]^{*}\right)\right) \\
& =H^{1}\left(X_{c}, \mathbf{K}_{X_{c}} \otimes\left[(r+1) \pi^{-1}(H) \cap X_{c}\right]\right) \\
& =H^{1}\left(X_{c}, \mathbf{K}_{X_{c}} \otimes\left(\left.\pi\right|_{X_{c}}\right)^{*}[(r+1) H]\right) .
\end{aligned}
$$

On the other hand since $[H]$ is a positive line bundle and $\left.\pi\right|_{X_{c}}$ is locally a biholomorphic map $\left(\left.\pi\right|_{X_{\mathrm{c}}}\right) *[H]$ is a positive line bundle over $X_{c}$. Hence by the Nakano's vanishing theorem (cf. [7], Theorem 1) we obtain

$$
H^{1}\left(X_{c}, \mathbf{K}_{X_{c}} \otimes\left(\left.\pi\right|_{X_{c}}\right) *[(r+1) H]\right)=0
$$

Thus $f$ can be extended to a holomorphic function $\tilde{f}$ on $X_{c}$. Since the choice of $x$ was arbitrary this implies that $X_{c}$ is holomorphically convex. Hence by Theorem $1.3 X$ is holomorphically convex. Thus by induction 
we obtain the theorem for $\mathbf{P}^{n}$. The proof for $\mathbf{Q}^{n}$ is similar. Q.E.D.

\section{Weakly 1-complete surface}

In this paragraph we prove the following

Theorem 2.2. Let $X$ be a weakly 1-complele manifold of dimension 2. If the canonical bundle of $X$ is negative, then $\mathrm{Y}$ is holomorphically convex.

Proof. Let $\psi$ be an exhaustion function on $X$. First we assume that there exists a point $x \in X$ where $\varphi$ is strictly plurisubharmonic. Let $\varpi: \widetilde{X} \rightarrow X$ be the blowing up of $X$ at $x$. Then as usual the obstruction for finding a holomorphic function on $X$ with a prescribed differential at $x$ lies in the group $H^{1}\left(X,\left[2 \varpi^{-1}(x)\right]^{*}\right)$. Note that $\varpi^{-1}(x) \cong \mathbb{P}^{1}$ and $\left.\operatorname{deg}\left[\varpi^{-1}(x)\right]\right|_{\varpi^{-1}(x)}=-1$, where we denote by $\operatorname{deg} L$ the degree of the line bundle $L$ over $\mathbf{P}^{1}$. We have

$$
\left.\operatorname{deg} \mathbf{K}_{\tilde{X}}\right|_{\varpi^{-1}(x)}=\operatorname{deg} \mathbf{K}_{\mathbf{p}^{1}}+\left.\operatorname{deg}\left[\varpi^{-1}(x)\right]^{*}\right|_{\varpi^{-1}(x)}=-1 .
$$

Hence $\left.\mathbf{K}_{\tilde{X}}^{*} \otimes\left[2 \varpi^{-1}(x)\right]^{*}\right|_{\Phi^{-1}(x)}$ is a positive line bundle over $\varpi^{-1}(x)$. So there exist neighbourhoods $W_{1}$ and $W_{2}$ of $\varpi^{-1}(x)$ such that $W_{1} \Subset W_{2}$, $\mathbf{K}_{\tilde{X}}^{*} \otimes\left[2 \varpi^{-1}(x)\right]^{*}$ is a positive line bundle over $W_{1} \cup\left(X-\bar{W}_{2}\right)^{*}$ and $\varphi \circ \varpi$ is strictly plurisubharmonic in a neighbourhood of $\bar{W}_{2}-W_{1}$. Multiplying $\exp \left(-\mu(\varphi \circ \varpi)\right.$ to the metric along the fibers of $\mathbf{K}_{\tilde{X}}^{*} \otimes\left[2 \varpi^{-1}(x)\right]^{*}$, where $" l$ is a sufficiently large positive number, we immediately see that $\mathbf{K}_{\tilde{X}}^{*} \otimes\left[2 \varpi^{-1}(x)\right]^{*}$ is a positive line bundle over $\widetilde{X}$. Thus by the Nakano's vanishing theorem we obtain $H^{1}\left(\widetilde{X},\left[2 \varpi^{-1}(x)\right]^{*}\right)=0$. So there exists a nonconstant holomorphic function on $X$. Hence by Proposition $1.4 X$ is holomorphically convex. Let us assume that $\exp \varphi$ is nowhere strictly plurisubharmonic on $X$. Since, by Sard's theorem, there exists a $c \in \mathbb{R}$ such that $d \varphi$ is nowhere zero on $\partial X_{c}$, replacing $\varphi$ by $\exp \varphi$ if necessary, we may assume that $\partial \bar{\partial} \varphi$ annihilates every holomorphic tangent vector of $\partial X_{c}$. In other words, letting $S$ be the subbundle of the complexified tangent bundle of $\partial X_{c}$ which consists of the holomorphic tangent vectors

*) Note that by the assumption $\mathbf{K}_{\tilde{X}}^{*} \otimes\left[2 \widetilde{\omega}^{-1}(x)\right]^{*}$ is positive over $\widetilde{X}-\bar{W}_{2}$. 
and $\bar{S}$ be the conjugate of $S, S \oplus \bar{S}$ is exactly the null space of $\partial \bar{\partial} \varphi$. It is easily verified that if a holomorphic tangent vector $\xi$ is annihilated by $\partial \bar{\partial} \varphi$, then the tangent vector $[\xi, \bar{\xi}]$ is also annihilated by $\partial \bar{\partial} \varphi$, where $[\xi, \bar{\xi}]$ is defined by extending $\xi$ as a local vector field. It follows that $[S, \bar{S}] \subset S \oplus \bar{S}$. This is the integrability condition of $S$ so that there exists a foliation (of class $C^{\infty}$ ) on $\partial X_{c}$ whose complexified tangent bundle is $S \oplus \bar{S}$. Let $\mathcal{L}$ be a maximal leaf of this foliation. Then $\mathcal{L}$ is a complex submanifold of $X$ since at every point of $\mathcal{L}$ the tangent space is a complex line in the tangent space of $X$ at that point. Since $\partial X_{c}$ is compact and the canonical bundle of $X$ is negative, there exists a complete hermitian metric on $\mathcal{L}$ whose Gaussian curvature is greater than some positive constant.*) Therefore by the theorem of Bonnet (cf. [10], Chapter 8, Theorem 17) $\mathcal{L}$ is compact. Since $\left.[\mathcal{L}]\right|_{\mathcal{L}}$ is the trivial bundle**) the canonical bundle of $\mathcal{L}$ is negative. Hence $\mathcal{L} \cong \mathbf{P}^{1}$ and there exists a neighbourhood $W$ of $\mathcal{L}$ and a proper holomorphic map $\delta$ from $W$ to an open disc $\Delta \subset \mathbf{C}$ such that $\delta$ is of maximal rank and $\delta^{-1}(0)=\mathcal{L}$ (cf. [5], Main theorem). To complete the proof we need the following.

Lemma. The line bundle $[m \mathcal{L}]$ is semi-positive for any integer $m$.

Admitting the lemma, we proceed as follows: By the Nakano's vanishing theorem we obtain

$$
H^{1}\left(X,[2 \mathcal{L}]^{*}\right)=H^{1}\left(X, \mathbf{K}_{X} \otimes \mathbf{K}_{X}^{*} \otimes[2 \mathcal{L}]^{*}\right)=0 .
$$

That $H^{1}\left(x,[2 \mathcal{L}]^{*}\right)=0$ implies that there exists a holomorphic function on $X$ with a prescribed differential along $\mathcal{L}$ so there exists a nonconstant holomorphic function on $X$. Thus by Proposition $1.4 X$ is holomorphically convex.

Proof of Lemma. Since $\left.\varphi\right|_{\delta^{-1}(t)}$ is constant for every $t \in \Delta, \varphi \circ \delta^{-1}$ is $C^{\infty}$ and subharmonic on $\Delta$. Since $\partial \bar{\partial} \varphi$ has no zero on $\partial X_{c}$, choosing 1 smaller if necessary, we may assume that $\varphi \circ \delta^{-1}$ is strictly subharmonic

*) **) See the appendix. 
on $\Delta$. Let $\Delta^{\prime}$ be a neighbourhood of 0 such that $\Delta^{\prime} \subseteq \Delta$. Let $\approx$ be the coordinate of $\mathbf{C}$. We choose a metric $\left\{a_{i}\right\}, i=1,2$ along the fibers of $[m \mathcal{L}]$ as follows:

$$
\begin{aligned}
& a_{1}: \delta^{-1}(\Delta) \rightarrow \mathbb{R}, \\
& a_{1}:=\rho \circ \delta,
\end{aligned}
$$

where $\rho(z)$ is a positive $C^{\infty}$ function such that $\rho(z)=|z|^{-2 n}$ on $d-d^{\prime}$.

$$
\begin{aligned}
& a_{2}: X-\delta^{-1}\left(J^{\prime}\right) \rightarrow \mathbb{R}, \\
& a_{2}:=1 .
\end{aligned}
$$

It is easy to see that $[m \mathcal{L}]$ is semipositive with respect to the metric $\left\{a_{i} \exp (-\nu \varphi)\right\}$ if $\nu$ is a sufficiently large positive number. Q.E.D.

\section{Complete intersection of type $(2.2)$}

Let $M \subset \mathbb{P}^{n}$ be a complex submanifold of codimension 2. $\quad M$ is called a complete intersection of type (2.2) if there exist hyperquadrics $\mathbb{Q}_{1}, \mathbb{Q}_{2} \subset \mathbb{P}^{n}$ such that $M=\mathbf{Q}_{1} \cap \mathbf{Q}_{2}$ in scheme theoretic sense.

Theorem 2. 3. Let $X$ be a weakly 1-complete manifold. If $X$ is a domain over a complete intersection of type (2.2), then $\mathrm{Y}$ is holomorphically convex.

Proof. Let $M$ be a complete intersection of type (2.2) and $X$ a weakly 1-complete manifold which is a domain over $M$. Since the theorem is well known if $\operatorname{dim} M=1$, we may assume $\operatorname{dim} M \geqq 2$. We prove the theorem by induction on $\operatorname{dim} M$. Since $\operatorname{dim} M \geqq 2$ the canonical bundle of $M$ is negative. Therefore if $\operatorname{dim} M=2$, by Theorem $2.2 \mathrm{X}$ is holomorphically convex. Let us assume that the theorem is valid if $\operatorname{dim} M \leqq n$, where $n \geqq 2$. Let $\operatorname{dim} M=n+1$ and $X_{c}$ a sublevel set rela. tive to an exhaustion function on $X$. We may assume that $\partial X_{c} \neq \emptyset$. Let $x \in \partial X_{c}$ and $\pi: X \rightarrow M$ be the given locally biholomorphic map. Then by Bertini's theorem there exists a hyperplane $H \subset \mathbf{P}^{n+3}$ which contains $\pi(x)$ and intersects $M$ transversally. We may assume that $H$ contains $\pi(y)$ for some $y \in X_{c}$. By the induction hypothesis $\pi^{-1}(H \cap M) \cap X_{c}$ is holomorphically convex. On the other hand 


$$
\begin{aligned}
& {\left[\pi^{-1}(H \cap M)\right]^{*}} \\
& \quad=\mathbf{K}_{X} \otimes \mathbf{K}_{X}^{*} \otimes\left[\pi^{-1}(H \cap M)\right]^{*} \\
& \quad=\mathbf{K}_{X} \otimes \pi^{*}\left(\left.\mathbf{K}_{M} \otimes[H]\right|_{M} ^{*}\right) \\
& \quad=\mathbf{K}_{X} \otimes \pi^{*}\left(\left.\left.[n H]\right|_{M} \otimes[H]\right|_{M} ^{*}\right) \\
& \quad=\mathbb{K}_{X} \otimes \pi^{*}\left(\left.[(n-1) H]\right|_{M}\right) .
\end{aligned}
$$

Since $n \geqq 2, \pi^{*}\left(\left.[(n-1) H]\right|_{M}\right)$ is a positive line bundle over $X$. Hence by the Nakano's vanishing theorem we obtain

$$
H^{1}\left(X_{c},\left.\left.\mathbf{K}_{X_{c}} \otimes \pi\right|_{X_{c}} ^{*}[(n-1) H]\right|_{M}\right)=0 .
$$

The remaining part of the proof is similar to that of Theorem 2.1.

Q.E.D.

Remark. Similarly as above one can prove the holomorphical convexity of weakly 1-complete domains over hypersurfaces of degree 3 .

\section{Appendix}

Let $M$ be a complex manifold of dimension one provided with a hermitian metric $d s^{2}=a(z) d z \cdot d \bar{z}$. The hermitian metric $d s^{2}$ is called complete if every geodesic ball is relatively compact. We define the Gaussian curvature $\rho(z)$ of $d s^{2}$ as follows:

$$
\rho(z):=-\frac{-1}{a(z)} \frac{\partial^{2} \log a(z)}{\partial z \partial \bar{z}}
$$

Proposition. Let $X$ be a weakly 1-complete manifold of dimension 2 with an exhaustion function $\varphi$. Assume that the canonical bundle $\mathbf{K}_{X}$ of $X$ is negative and $\varphi$ is nowhere strictly plurisubharmonic. Let $c$ be a noncritical value of $\varphi$. Let $\mathfrak{X}_{c}$ be the foliation (of class $C^{\infty}$ ) whose tangent bundle is the nullity of $\partial \bar{\partial} \varphi$. Then every maximal leaf of $\mathfrak{X}_{c}$ is provided reith a complete hermitian metric whose Gaussian curvature is greater than some positive constant.

Proof. Let $\left\{W_{i}\right\}$ be a locally finite trivializing covering of $\mathbf{K}_{Y}$ and denote by $\left\{a_{i}\right\}$ the metric along the fibers of $\mathbf{K}_{X}$ such that 
$-\sqrt{-1} \partial \bar{\partial} \log a_{i}$ is a negative $(1,1)$ form on $X$. We may assume that there exists a system of complex coordinates $\left(z_{i}, t_{i}\right)$ on $W_{i}$ such that the norm of $d z_{i} \wedge d t_{i}$ is $\sqrt{a_{i}}$ and $z_{i}$ gives a local coordinate when it is restricted to the leaves of $\mathscr{X}_{c}$. Let $\mathcal{L}$ be a maximal leaf and $\left\{w_{j}\right\}$ be a set of local defining equations of $\mathcal{S}$ associated to an open covering $\left\{U_{j}\right\}$ of $X$ such that for every $j$ there exists $W_{\nu(j)}$ such that $U_{j} \subset W_{\nu(j)}$. Letting $\left(z_{\nu(j)}, w_{j}\right)$ be a local coordinate in a neighbourhood of $U_{j}$, we let

$$
u_{j}:=\frac{\partial \varphi}{\partial w_{j}^{\prime} w_{j=0}} .
$$

It is clear (and also the point) that $\iota_{j}$ are nonvanishing holomorphic functions. Therefore the normal bundle of $\mathcal{L}$ in $X$ is trivial. Taking a refinement of $\left\{U_{j}\right\}$ and multiplying $w_{j}$ by constants if necessary, we may assume that

$$
1<\left|u_{j}\right|<2
$$

We obtain a hermitian metric defined by

$$
d s^{2}=\left.a_{\nu(j)}\left|u_{j}\right|^{2}\left|\frac{d t_{\nu(j)}}{d w_{j}}\right|_{w_{j}=0}\right|^{-2} d z_{\nu(j)} \cdot d \bar{z}_{\nu(j)}
$$

on $\mathcal{L}$, where we identify $z_{\nu(j)}$ as a local coordinate of $r$. Since $2>\left|u_{j}\right|>1, d s^{2}$ is complete and its Gaussian curvature is greater than some positive constant.

Q.E.D.

\section{References}

[1] Grauert, H., Bemerkenswerte pseudokonvexe Mannigfaltigkeiten, Miath. Z., 81 (1963), 377-391.

[2] Gunning, R. C. and Rossi, H., Analytic functions of several complex variables, Engelwood Cliffs, N. J. Prentice-Hall, 1965.

[3] Hirschowitz, A., Le problème de Levi pour les espaces homogènes, Bull. Soc. Math. France, 103 (1975), 191-201.

[4] Knorr, K. and Schneider, M., Relativexzeptionelle analytische Mengen, Math. Ann., 193 (1971), 238-254.

[5] Kodaira, K., A theorem of completeness of characteristic systems for analytic families of compact submanifolds of complex manifolds, Ann. of Math., 75 (1962), 146-162.

[6] Narashimhan, R., The Levi problem for complex spaces 11, Math. Ann., 146 (1962), 195-216.

[7] Nakano, S., Vanishing theorems for weakly 1-complete manifolds II, Publ. RIMS, 
Kyoto Univ., 10 (1974), 101-110.

[8] Richberg, R., Stetige streng pseudokonvexe Funktionen, Math. Ann., 175 (1968), 257-286.

[9] Siu, Y. T., Pseudoconvexity and the problem of Levi, Bull. Amer. Math. Soc., 84 (1978), 481-512.

[10] Spivak, M., Differential geometry, 4, Publish or Perish Inc., 1975. 